\title{
Stem Cell Transplantation in Brain Tumors: A New Field for Molecular Imaging?
}

\author{
Nora Sand $u^{1}$ and Bernhard Schaller ${ }^{2}$ \\ ${ }^{1}$ Department of Neurosurgery, University of Lausanne, Lausanne, Switzerland; ${ }^{2}$ Department of Neurosurgery, University of Paris, \\ Paris, France
}

\begin{abstract}
Neural stem cells have been proposed as a new and promising treatment modality in various pathologies of the central nervous system, including malignant brain tumors. However, the underlying mechanism by which neural stem cells target tumor areas remains elusive. Monitoring of these cells is currently done by use of various modes of molecular imaging, such as optical imaging, magnetic resonance imaging and positron emission tomography, which is a novel technology for visualizing metabolism and signal transduction to gene expression. In this new context, the microenvironment of (malignant) brain tumors and the blood-brain barrier gains increased interest. The authors of this review give a unique overview of the current molecular-imaging techniques used in different therapeutic experimental brain tumor models in relation to neural stem cells. Such methods for molecular imaging of gene-engineered neural stem/progenitor cells are currently used to trace the location and temporal level of expression of therapeutic and endogenous genes in malignant brain tumors, closing the gap between in vitro and in vivo integrative biology of disease in neural stem cell transplantation.
\end{abstract} (C) 2010 The Feinstein Institute for Medical Research, www.feinsteininstitute.org Online address: http://www.molmed.org doi: $10.2119 / \mathrm{molmed} .2010 .00035$

\section{INTRODUCTION}

Neural stem cells (NSCs) have the tremendous potential to migrate to areas of pathology in the central nervous system (CNS) $(1,2)$. When implanted into diseased or injured CNS, NSCs can travel great distances and engraft within discrete areas as well as diffuse neuronal abnormalities $(1,2)$. Engraftment is often followed by integration into the local neural milieu, accompanied by stable gene expression from the NSCs $(1,2)$. In addition, the pluripotency of NSCs endows them with the capability to replace diseased CNS tissues in an appropriate manner $(1,2)$. Recent evidence has also suggested that engrafted exogenous NSCs may have effects on the surrounding microenvironment, such as promoting neuroprotection and / or regeneration of host neural pathways (1-4). These characteristics of NSCs make them ideal agents for the treatment of various CNS pathologies, especially brain tumors $(1,2)$.

\section{BIOLOGICAL USE OF NEURAL STEM CELLS FOR BRAIN TUMOR THERAPY}

Brain tumors are generally difficult to treat because of the unique neuroanatomical location of the lesions next to critical neurovascular structures $(1,5,6)$. In addition, the extensive infiltrative nature of the tumor cells makes their effective and total eradication challenging. These difficulties are reflected in the high rate of treatment failure and disease recurrence (1). In addition, normal brain structures are distorted and often destroyed by the growing neoplasm (1). Even with effective therapy to surgically resect and destroy the neoplastic tissues, the brain is still injured, which often leaves the patient in a debilitated state $(1,3,4)$.

The inherent tumor tropism of NSCs to primary and invasive tumor foci can be exploited to deliver therapeutic agents to invasive brain tumor cells in humans $(1,2)$. NSCs have tremendous potential to migrate to the pathological brain areas. When implanted into a diseased or injured nervous system, NSCs can travel great distances to, and engraft within, target areas (1). Engraftment is often followed by integration into the local neural milieu, accompanied by stable gene expression from the NSCs $(1,7)$. The use of such a strategy to convert prodrug to drug via therapeutic transgenes delivered by immortalized therapeutic NSC lines has shown efficacy in animal models $(1,2)$. In addition, the pluripotency of NSCs endows them with the capability to replace diseased (neural) tissues in an appropriate manner. Recent evidence has also suggested that engrafted exogenous NSCs may have effects on the surrounding microenvironment, such as promoting protection and/or regeneration of host neural pathways $(1,7)$. These characteristics of NSCs may make them ideal agents for the treatment of brain tumors $(1,2)$.

\section{THE MICROENVIRONMENT OF BRAIN TUMORS}

The pathological characteristics of malignant brain tumors are exemplified by active invasiveness, necrosis and a spe-
Address correspondence and reprint requests to Bernhard Schaller, University of Oradea, Romania. E-mail: bernhard.schaller@yahoo.de. Submitted March 17, 2010; Accepted for publication June 28, 2010; Epub (www.molmed.org) ahead of print June 30, 2010. 
cialized form of angiogenesis, known as microvascular hyperplasia. Such pathological features are thought to be due to tissue hypoxia. Therefore hypoxia is a critical aspect of the surrounding microenvironment of brain tumors and is generally associated with unfavorable clinical outcomes (3,4,8-12). Cells that are under hypoxic stress can develop an adaptive response that includes increased rates of glycolysis and angiogenesis or undergo cell death by promoting apoptosis and/or necrosis $(3,8,13)$. The ability of tumor cells to maintain a balance between adaptation to hypoxia and cell death is regulated by hypoxiainducing factors, a family of transcription factors that are essential for the regulation of the expression of a large number of hypoxia-responsive genes $(13,14)$. Tumor hypoxia is hypothesized to facilitate metastases, tumor recurrence, invasive potential and resistance to chemotherapy and radiotherapy, which culminate in decreased patient survival. For this reason, effective targeting of hypoxic areas in brain tumors remains a significant therapeutic challenge $(3,4,8-10)$. New NSC therapeutic options for tumor-targeted drug delivery show promise in treatment of brain tumors that are refractory to traditional therapies $(1,3,4,9)$. However, the molecular mechanisms of NSC-targeting to hypoxic tumor areas are not well understood $(3,4,9)$. The unique ability of NSCs to "home in" on tumor cells and then deliver a desired gene product makes NSCs a promising agent in brain tumor therapy (1). Cytolytic viruses and genes coding for antitumor cytokines, prodrug-converting enzymes and various neurotrophic factors have all been engineered into engraftable NSCs for delivery to tumors (1). Novel brain tumor treatment strategies that involve transplantation or infusion of cells that seek out invading tumor cells demand thorough in vivo monitoring $(3-8,15,16)$. In particular, NSCs have attracted great interest because they have demonstrated tropism to tumor cells and even long-distance migration to single tumor cells $(17,18)$. It is thought that the

Table 1. Representative examples of different molecular-imaging modalities in experimental brain tumor models related to neural stem cells.

\begin{tabular}{|c|c|c|c|c|}
\hline Reference & Imaging & Model & Stem cell & Monitoring \\
\hline Hata et al. (57), 2010 & Optical & Clone & Mesenchymal & Incorporation \\
\hline Shah et al. (54), 2008 & Optical & Mouse & Neural & Migration, survival \\
\hline Brekke et al. (55), 2007 & MRI & Rat & Neural & $\begin{array}{l}\text { Migration, therapeutic } \\
\text { efficacy }\end{array}$ \\
\hline Anderson et al. (56), 2005 & MRI & Mouse & Bone marrow & Incorporation \\
\hline Miletec et al. (58), 2007 & PET/MRI & Rat & Mesenchymal & Therapeutic efficacy \\
\hline
\end{tabular}

migration of NSCs to neoplastic cells is mediated by the secretion of chemical factors, such as vascular endothelial growth factor (19), that are involved in the proliferation, growth and maintenance of tumors. Transplantation of unaltered NSCs has resulted in prolonged survival of animals with experimentally induced tumors (20), and the insertion of antitumor cytokine genes (for example, $I L-12$ ) or proapoptotic genes (for example, TRAIL) has further improved the efficiency of this approach (21). Little is known about how these cells exert their beneficial effects in vivo. Despite contrary evidence from preclinical studies, there is some concern that transplantation of stem cells could further exacerbate tumor formation. This is caused by mounting evidence that brain tumors may be caused by a single NSC that did not differentiate (22). The ability to monitor cell therapy in vivo is therefore desirable because it may provide more control over the activity of NSCs (23). One possibility is that stem cells will be engineered with a suicide gene (24) that could be activated if transplanted cells did not behave in a therapeutic manner.

\section{THE PROBLEM OF THE BLOOD-BRAIN BARRIER}

One hallmark of malignant brain tumors is the disruption of the normal homeostasis between angiogenic and antiangiogenic factors. The resulting vasculature is characterized by tortuous vessels that feature disruptions in their structural integrity, increased leakiness, uneven focal thickness and arteriovenous shunts not seen in the normal brain vasculature as well as changes in the physiology of the blood-brain barrier (BBB).
However, brain delivery of therapeutic cells is physiologically limited by the $\mathrm{BBB}$, which remains one of the recognized rate-limiting steps $(25,26)$. As the BBB limitation has been more and more acknowledged, many innovative surgical and pharmacological strategies have been developed to circumvent it. Since opening of the BBB was first reported by Rapoport et al. in 1972 (27), preclinical studies have provided important information on the extent of BBB permeation. Although the BBB is frequently leaky in the center of malignant brain tumors, the well-vascularized actively proliferating edge of the tumor has been shown to have variable and complex barrier integrity (27). Current experimental data show that brain penetration of peripherally circulating cells, such as stem cells and immune cells targeting the CNS, requires BBB disruption and is limited to the immediate perivascular space. In addition, oncolytic virus treatment of rat gliomas has been shown to be associated with a significant increase in the permeability of the tumor vasculature and consequently with significant increases in tumor inflammation and leukocyte infiltration (28).

\section{IMAGING MODALITIES}

Molecular imaging has given investigators a high-throughput, inexpensive and sensitive means for tracking in vivo cell proliferation for periods of days, weeks, and even months $(29,30)$ (Table 1$)$. This advancement has led to significantly increased understanding of the spatiotemporal kinetics of NSC engraftment, localization, viability and proliferation in living subjects (29-31). A major advance in molecular imaging has been 
the extension of noninvasive reporter gene assays from molecular and cellular biology into in vivo multimodality imaging platforms $(15,29,30)$. The ability to control the activity of transplanted cells presupposes that it is possible to visualize the presence, location and activity of stem cells in vivo. An innovative approach to harnessing the respective strengths of various imaging platforms is the creation and use of a fusion reporter construct (31). These reporter genes, under control of engineered promoters and enhancers that take advantage of host-cell transcriptional machinery, are introduced into cells by using a variety of vector and nonvector methods (5). Once in the cell, reporter genes can be transcribed either constitutively or only under specific biological or cellular conditions, depending on the type of promoter used $(29,30,32)$. Transcription and translation of reporter genes into bioactive proteins is then detected with sensitive, noninvasive instrumentation (for example, charge-coupling device cameras) by using signal-generating probes such as D-luciferin for optical imaging (30). To avoid the need for excitatory light to track stem cells in vivo, as is required for fluorescence imaging, bioluminescence reporter gene imaging systems require only an exogenously administered probe to induce light emission that can be monitored by optical imaging (30). Stably transduced cells that carry the reporter construct within their chromosomal DNA will pass the reporter construct DNA to daughter cells, allowing for longitudinal monitoring of NSC survival and proliferation in vivo. However, the clinical utility of these imaging modalities is limited by poor tissue penetration and low spatial resolution, making them impractical for use in patient trials. In the context of NSC-based therapies, magnetic resonance imaging (MRI) can be used to noninvasively follow dynamic spatiotemporal patterns of NSC tumor targeting, allowing for the optimization of treatment strategies, and to assess the efficacy of the therapy (33). Clinical MRI at 3 Tesla, however, has high spatial resolution with excellent soft tissue contrast for noninvasive, dynamic in vivo assessment of cellular trafficking at multiple time points (33). Research on MRI visualization of cellular tracking is rapidly expanding, and many studies have been published during the last decade. Relevant studies include mouseand rat-derived stem and progenitor cells transplanted into the brain, spinal cord or vasculature of laboratory animals by using strongly T1-weighted paramagnetic contrast labels such as gadolinium $(34,35)$, and the use of T2- and T2*weighted superparamagnetic iron oxide nanoparticles (36). Although MRI has been extensively used to track cells repeatedly in vivo (37), the higher specificity of positron emission tomography (PET) ligands and the ability of PET to detect reporter genes are promising features for the development of long-term in vivo monitoring of transplanted cells. Such molecular imaging has expanded the role of PET from the research domain into clinical application for neurosciences $(6,32,38)$. Currently, PET is being used as a clinical molecular-imaging tool in neurosurgery. For cellular imaging by MRI, the contrast agents used to detect transplanted cells from the background of the brain are incorporated into the cell in vitro before transplantation. The presence of the contrast agent inside the cell therefore allows the prolonged visualization of grafted cells on MRI scans. However, the continued presence of the contrast agent inside the cell can also affect cellular functions (37). Although this approach has been demonstrated with PET/ single-photon emission computed tomography ligands and has allowed monitoring for up to 14 days (39), the short half-life of PET ligands compromises the long-term visualization of cells. A more promising method is to engineer reporter genes inside the cells before grafting and to systemically inject the PET ligand to detect transplanted cells (40). The use of reporter genes avoids most issues pertaining to the long-term effects the contrast agent might exert onto cellular functions. The repeated application of a contrast agent complements a flexible imaging approach that can target various relevant targets, such as 2-deoxy-2[(18)F]fluoro-Dglucose (FDG)-PET, used to investigate tumor metabolism. An important mechanism to transport FDG into the transformed cell is based on the action of glucose transporter proteins; furthermore, highly active hexokinase bound to tumor mitochondria helps to trap FDG in the cell. In addition, enhanced FDG uptake may be due to relative hypoxia in brain tumor masses, which activates the anaerobic glycolytic pathway (41). For continued assessment of tumor evaluation in response to treatment, it is important to be able to assess the malignancy of the tumor noninvasively at various time points, and thus it seems preferable to use quantitative instead of semiquantitative FDG-PET assessment of tumor metabolism $(42,43)$. The main concerns with this approach involve the potential immunogenic properties of reporter genes, which might lead to graft rejection or the downregulation of the reporter gene and thus abolish graft detection. Unfortunately, at present little research is dedicated to the development of PET techniques that would allow the continued assessment of cell therapy. The use of radioligands to a reporter gene will provide maximum flexibility and further integration into a wider molecular imaging strategy of brain tumors. Apart from in vivo monitoring, PET will also be instrumental in enabling investigators to gain a greater mechanistic understanding of how cell therapy exerts its therapeutic effects. Because stem cells can be engineered to express particular genes and serve as delivery vehicles (44), they may affect tumor cells through inhibition of angiogenesis or induction of tumor cell apoptosis or differentiation. The ability to study how stem cells alter the molecular composition of the tumor will allow in vivo monitoring of the therapeutic efficacy of cell therapy. In addition, PET imaging allows the serial in vivo assessment of the inflammatory and immunological response 
through visualization of, for instance, a T-cell response (45). Modulation of the immune response could provide additional benefits to combat tumor cells. PET imaging is at present the only in vivo technology that can be used to assess these various molecular aspects of stem cell efficacy and lead to a mechanistic understanding of how stem cells attack tumor cells. PET is already used clinically to evaluate stem cell therapy for lymphoma. Simple FDG-PET leads to a $92 \%$ positive and $88 \%$ negative prediction of treatment outcome and correlates more strongly with disease-free survival than computer-assisted tomography (46). FDG-PET can therefore already play an important role in precise patient selection and help investigators to determine which conditions are most suitable for stem cell therapy $(47,48)$. Serial quantitative PET will provide a means to monitor treatment progression (49) and afford a refinement of dosage and integration with other therapies. Imaging of brain tumors already commonly uses FDG-PET. Translation of cell therapy for brain tumors will be best suited for use in conditions in which imaging technology can already provide a robust baseline assessment that would allow careful patient selection. As the clinical translation of stem cell therapy for brain tumors progresses (49), preclinical validation studies will need to rely more on molecular imaging to ensure the safety and efficacy of this promising therapy. The growing sophistication of molecular imaging will allow increasingly sophisticated therapeutic approaches to be visualized and monitored in vivo (50-53). The efficacy of the multitude of therapeutic effects that can be exerted by unaltered and genetically engineered stem cells must be determined in relation to the molecular composition of the tumor. The ability to select patients at an early stage based on a molecular signature of the entire tumor will allow the clinicians to determine what strategy might be best suited to remove the neoplastic cells. Although PET is commonly used in preclinical and clinical studies for visualization of various tumors and drug interactions and for understanding tumor metabolism with high specificity, the relatively low spatial resolution of PET and its radiation dose and relatively short-term signal production make it a nonideal technique for clinical tracking of cells to tumors, which requires extended periods of observation. Further developments in molecular imaging may provide the diagnostic framework for the use of molecular medicine to treat brain tumors (6).

\section{FROM BENCH TO BEDSIDE}

Current investigations of the use of monitoring by molecular imaging are increasing our understanding of stem cells and stem-cell regulation, which in turn will facilitate the development of novel therapies to eliminate (malignant) brain tumors. Given the current status of therapeutic developments in neurooncology, we can expect a number of drug targets to emerge that can be exploited by means of interstitial or intracavitary delivery, are not neurotoxic, and may even be imaged in action with new molecular imaging modalities (59). For effective therapy for (malignant) brain tumors, convection-enhanced delivery, conditional replication of oncolytic viruses and motile, genetically engineered neural stem cells all seem to fulfill the distribution requirements needed to overcome the very limited efficacy offered by surgery, conventional chemotherapy and radiation treatments. Although these genomics-based discovery approaches are not specific for neurooncological targets, the development of delivery strategies is highly specific for the CNS, thus creating a unique set of organ- and diseasespecific therapies.

\section{DISCLOSURE}

The authors declare that they have no competing interests as defined by Molecular Medicine, or other interests that might be perceived to influence the results and discussion reported in this paper.

\section{REFERENCES}

1. Yip S, et al. (2003) Neural stem cell biology may be well suited for improving brain-tumor therapies. Cancer. J. 9:189-204.

2. Thu MS, et al. (2009) Iron labelling and preclinical MRI visualization of therapeutic human neural stem cell in a murine gliome model. PLoSOne. 4:e7218.

3. Schaller B. (2003) Neuroprotection in brain tumors: good sense or nonsense from the pathophysiological viewpoint? Nervenarzt. 74:134-6.

4. Schaller BJ, Buchfelder M. (2006) Neuroprotection in primary brain tumors: sense or nonsense? Expert Rev. Neurother. 6:723-30.

5. Schaller BJ, Cornelius JF, Sandu N, Buchfelder M. (2008) Molecular imaging of brain tumors: Personal experience and review of the literature. Curr. Mol. Med. 8:711-26.

6. Schaller B, Cornelius JF, Sandu N. (2008) Molecular medicine successes in neuroscience. Mol. Med. 14:361-4.

7. Kosztowski T, Zaidi HA, Quinones-Hinojasa A. (2009) Application of neural and mesenchymal stem cells in the treatment of gliomas. Expert Rev. Anticancer Ther. 9:597-612.

8. Schaller B. (2005) Influences of brain tumor-associated $\mathrm{pH}$ changes and hypoxia on epileptogenesis. Acta Neurol. Scand. 111:75-83.

9. Zhao P, et al. (2008) Neural stem cell tropism to glioma: critical role of tumor hypoxia. Mol. Cancer Res. 6:1819-29.

10. Matuski E, et al. (2009) Cell adhesion markers in ischaemic stroke patients: correlation with clinical outcome and comparison with primary autoimmune disease. Arch. Med. Sci. 5:182-9.

11. Schaller BJ. (2006) The role of endothelin in stroke: experimental data and underlying pathophysiology. Arch. Med. Sci. 2:146-58.

12. Schaller B. (2005) Ischemic preconditioning as induction of ischemic tolerance after transient ischemic attacks in human brain: its clinical relevance. Neurosci. Let. 377:206-11.

13. Oliver L, Olivier C, Marhuenda FB, Campone M, Vallette FM. (2009) Hypoxia and the malignant glioma microenvironment: regulation and implications for therapy. Curr. Mol. Pharmacol. 2:263-84.

14. Schaller B, Graf R. (2004) Cerebral ischemia and reperfusion: the pathophysiologic concept as a basis for clinical therapy. J. Cereb. Blood Flow Metab. 24:351-71.

15. Schaller BJ, Modo M, Buchfelder M. (2007) Molecular imaging of brain tumors: A bridge between clinical and molecular medicine? Mol. Imaging Biol. 9:60-71.

16. Schaller B, Cornelius JF, Sandu N, Ottaviani G, Perez-Pinzon MA. (2009) Oxygen-conserving reflexes of the brain: the current molecular knowledge. J. Cell Mol. Med. 13:644-7.

17. Aboody K, et al. (2000) Neural stem cells display extensive tropism for pathology in adult brain: Evidence from intracranial gliomas. Proc. Natl. Acad. Sci. U. S. A. 97:12846-51.

18. Benedetti S, et al. (2000) Gene therapy of experi- 
mental brain tumors using neural progenitor cells. Nat. Med. 6:447-50.

19. Schmidt NO, et al. (2005) Brain tumor tropism of transplanted human neural stem cells is induced by vascular endothelial growth factor. Neoplasia. 7:623-9.

20. Staflin K, et al. (2004) Neural progenitor cell lines rat inhibit tumor growth in vivo. Cancer Res. 64:5347-54.

21. Ethesham M, et al. (2002) The use of interleukin 12-secreting neural stem cells for the treatment of intracranial glioma. Cancer Res. 62:5657-63.

22. Fomchenko EI, Holland EC. (2005) Stem cells and brain cancer. Exp. Cell Res. 306:323-9.

23. Modo M, Roberts TJ, Sandhu JK, Williams SCR. (2004) In vivo monitoring of cellular transplants by magnetic resonance imaging and positron emission tomography. Expert Opin. Bio. Ther. 4:145-55.

24. Zlokovic BV, Apuzzo ML. (1997) Cellular and molecular neurosurgery: Pathways from concept to reality, part 1: target disorders and concept approaches to gene therapy of the central nervous system. Neurosurgery. 40:789-803.

25. Marchi N, et al. (2010) Multimodal investigations of trans-endothelial cell trafficking under condition of disrupted blood-brain barrier integrity. BMC Neurosci. 9:11-34.

26. Gera A, Steinberg GK, Guzman R. (2010) In vivo neural stem cell imaging: Current modalities and future directions. Regen. Med. 5:73-86.

27. Rapoport SI, Hori M, Klatzo I. (1972) Testing of a hypothesis for osmotic opening of the bloodbrain barrier. Am. J. Physiol. 223:323-31.

28. Kurozumi K, et al. (2007) Effect of tumor microenvironment modulation on the efficacy of oncolytic virus therapy. J. Natl. Cancer Inst. 99:1768-81.

29. Bradbury MS, et al. (2007) Optical bioluminescence imaging of human ES cell progeny in the rodent CNS. J. Neurochem. 102:2029-39.

30. Wilson K, Yu J, Lee A, Wu JC. (2008) In vitro and in vivo bioluminescence reporter gene imaging of human embryonic stem cells. J. Vis. Exp. (14):740.

31. Narsinh KH, Cao F, Wu JC. (2009) Molecular imaging of human embryonic stem cells. Methods Mol. Biol. 515:13-32.

32. Jacobs AH, et al. (2003) PET-based molecular imaging in neuroscience. Eur. J. Nucl. Med. Mol. Imaging. 30:1051-65.

33. Thu MS, et al. (2009) Iron labeling and preclinical MRI visualization of therapeutic human neural stem cells in a murine glioma model. PLoS. 4:e7218.

34. Modo M, et al. (2002) Tracking transplanted stem cell migration using bifunctional, contrast agentenhanced, magnetic resonance imaging. Neuroimage. 17:803-11.

35. Modo M, et al. (2004) Mapping transplanted stem cell migration after a stroke: a serial, in vivo magnetic resonance imaging study. Neuroimage. 21:311-7.
36. Corot C, Robert P, Idee JM, Port M. (2006) Recent advances in iron oxide nanocrystal technology for medical imaging. Adv. Drug Deliv. Rev. 58:1471-504.

37. Modo M, Hoehn M, Bulte J. (2005) Cellular MR imaging. Mol. Imaging. 4:1Y21.

38. Schaller BJ. (2008) Strategies for molecular imaging dementia and neurodegenerative diseases. Neuropsychiatr. Dis. Treat. 4:585-612.

39. Chin BB, et al. (2003) 111In oxine labeled mesenchymal stem cell SPECT after intravenous administration in myocardial infarction. Nucl. Med. Commun. 24:1149-54.

40. Jacobs A, et al. (2001) Quantitative kinetics of (124I)FIAU in cat and man. J. Nucl. Med. 42:467-75.

41. Pauwels EK, et al. (1998) FDG accumulation and tumor biology. Nucl. Med. Biol. 25:317-22.

42. Weber WA, Schwaiger M, Avril N. (2007) Quantitative assessment of tumor metabolism using FDG-PET imaging. Nucl. Med. Biol. 27:683-7.

43. Schaller B. (2004) Usefulness of positron emission tomography in diagnosis and treatment followup of brain tumors. Neurobiol. Dis. 15:437-48.

44. Shah K, Hsich G, Breakefield XO. (2004) Neural precursor cells and their role in neuro-oncology. Dev. Neurosci. 26:118-30.

45. Koehne G, et al. (2003) Serial in vivo imaging of targeted migration of human HSV-TK-transduced antigen-specific lymphocytes. Nat. Biotechnol. 21:405-13.

46. Filmont JE, et al. (2003) Value of F-18 fluorodeoxyglucose positron emission tomography for predicting the clinical outcome of patients with aggressive lymphoma prior to and after autologous stem-cell transplantation. Chest. 124:608-13.

47. Becherer A, et al. (2002) Positron emission tomography with [18F]2-fluoro-D-2-deoxyglucose (FDG-PET) predicts relapse of malignant lymphoma after high-dose therapy with stem cell transplantation. Leukemia. 16:260-7.

48. Spaepen K, et al. (2003) Prognostic value of pretransplantation positron emission tomography using fluorine 18-fluorodeoxyglucose in patients with aggressive lymphoma treated with highdose chemotherapy and stem cell transplantation. Blood. 102:53-9.

49. Cremerius U, et al. (2002) Pre-transplant positron emission tomography (PET) using fluorine-18fluoro-deoxyglucose (FDG) predicts outcome in patients treated with high-dose chemotherapy and autologous stem cell transplantation for nonHodgkins lymphoma. Bone Marrow Transplant. 30:103-11.

50. Su H, Forbes A, Gamghir SS, Braun J. (2004) Quantization of cell number by a positron emission tomography reporter gene strategy. Mol. Imaging Biol. 6:139-48.

51. Brower V. (2005) Search and destroy: recent research exploits adult stem cells' attraction to cancer. J. Natl. Cancer Inst. 97:414-6.

52. De Witte O, et al. (2000) FDG-PET as a prognostic factor in high-grade astrocytoma. J. Neurooncol. 49:157-63.
53. Padma MV, et al. (2003) Prediction of pathology and survival by FDG PET in gliomas. J. Neurooncol. 64:227-37.

54. Shah K, et al. (2008) Biomodal viral vectors and in vivo imaging reveal the fate of human neural stem cells in experimental glioma model. J. Neurosci. 28:4406-13.

55. Brekke C, et al. (2007) Cellular multiparametric MRI of neural stem cell therapy in a rat glioma model. Neuroimage. 37:769-82.

56. Anderson SA, et al. (2005) Noninvasive MR imaging of magnetically labelled stem cells to directly identify neovasculature in a glioma model. Blood. 105:420-5.

57. Hata N, et al. (2010) Platelet-derived growth factor BB mediates the tropism of human mesenchymal stem cells for malignant gliomas. Neurosurgery. 66:144-56.

58. Miletic H, et al. (2007) Bystander killing of malig nant glioma by bone marrow-derived tumor-infiltrating progenitor cells expressing a suicide gene. Mol. Ther. 15:1373-81.

59. Shah K, Weissleder R. (2005) Molecular optical imaging: applications leading to the development of present day therapeutics. NeuroRx. 2:215-25. 